\title{
ADVANCED KERATOCONUS WITH LOW REFRACTIVE ERROR
}

\author{
Mounir, A. ${ }^{(*)} \&$ Mostafa, E. \\ ${ }^{1}$ Ophthalmology dept., Faculty of Medicine, Sohag Univ., Sohag, Egypt \\ ${ }^{(*)}$ E-mail: dramrmonir@yahoo.com
}

\begin{abstract}
In this report we are presenting a 28-year-old female with right keratoconus grade3 and left keratoconus grade 2 with low degree refraction (spherical equivalent in right eye $=-2.25$ and $+0.75 D$ in left eye). Axial length measurement revealed short axial length ( $R E=19.63 \mathrm{~mm}$, $L E=19.97 \mathrm{~mm}$ ). After counseling the patient, the decision was to go for right simultaneous femtosecond intracorneal ring segments implantation with corneal collagen cross linking. UCVA, $B C V A$, sphere, cylinder, spherical equivalent, RMS total high order aberrations(HOA), RMS coma, and RMS astigmatism were measured pre-and postoperatively in the first week, 1 month, 3 month, 6 month. The results were favorable as regards improvement in UCVA, BCVA, with mild hyperopic shift in the sphere as expected with decrease in the cylinder, and induced flattening. As for HOA, coma and astigmatism; there was marked decrease.
\end{abstract}

Keywords: Keratoconus, low refractive error, Hyperopia, Short axial length globe, Intracorneal ring segments, High order corneal aberrations

\section{Introduction}

Keratoconus is a progressive corneal deformation which causes changes in corneal shape that lead to impairment of visual acuity as a result of irregular astigmatism, progressive myopia, higherorder aberrations and corneal thinning [1]. Keratoconus is mostly associated with myopia. Hyperopic keratoconus is rare, yet patients with Down syndrome have a higher

\section{Case History}

A 28 years old lady presented for glasses prescription. In the last two years she was fitted three times with glasses (all prescriptions were lost); all of which incidence of hyperopia, and occasionally keratoconus [2]. Intracorneal ring segments (ICRSs) have been used to regularize the corneal shape and reduce corneal astigmatism along with spherical equivalence and higher order aberrations, improve visual acuity to acceptable limits [3-5]. In this article we report a case of advanced keratoconus with low refractive error.

were not optimum and left the patient dissatisfied. Before these two years she had no refractive correction. The uncorrected visual acuity (UCVA) in the right 
eye (RE) was 1 LogMAR corrected to 0.6 with a refraction of $-0.50 \mathrm{Ds}$ to $-3.50 \mathrm{Dc}$ x58 while the UCVA in the left eye (LE) was 0.6 LogMAR corrected to 0.3 with a refraction of $+1.75 \mathrm{Ds}$ to $-2.00 \mathrm{Dc}$ $x 095$. Both corneas were clear on slit lamp biomicroscopy with Vogt striae in the RE. No history of contact lens use was reported. She had normal phenotype and mental state. Her family medical history was irrelevant with no systemic or ocular illness in the family. Corneal topography was performed by Sirius Scheimpflug Analyzer (CSO, Florence, Italy). Simulated keratometry was $52.59 / 55.20 \mathrm{D}$ at $137^{\circ}$ in the RE $49.93 / 50.68 \mathrm{D}$ at $51^{\circ}$ in the LE. The posterior elevation map showed 45 $\mu \mathrm{m}$ of elevation in the RE and $45 \mu \mathrm{m}$ of elevation in the LE at the thinnest point. The excessive anterior and posterior elevation is a consistent and reliable finding in diagnosis of keratoconus. The thinnest point was $450 \mu \mathrm{m}$ in the RE and $435 \mu \mathrm{m}$ in the LE. The Sirius corneal topography findings indicated keratoconus grade 3 keratoconus in RE and Keratoconus grade 2 in LE, fig (1).

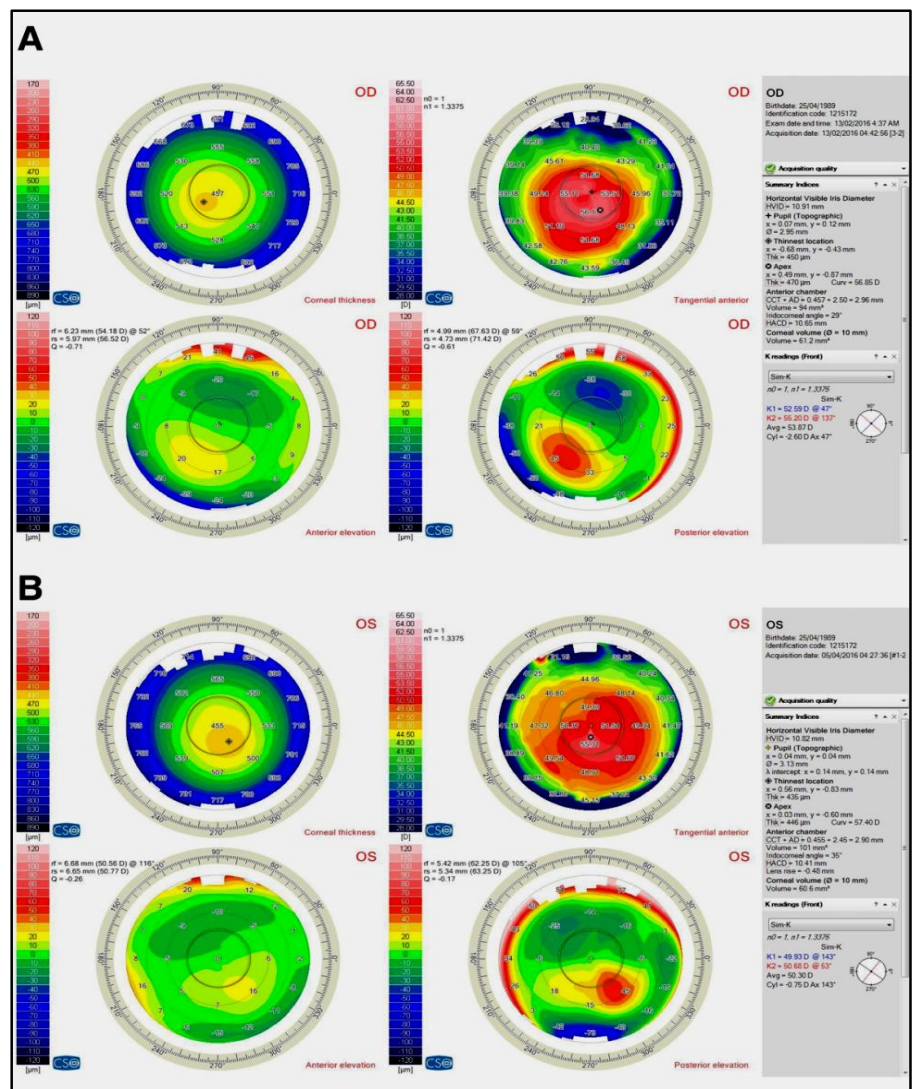

Figure (1) corneal topography of both eyes; $\underline{\mathbf{A}}$. RT. Eye, $\underline{\mathbf{B}}$. LT. Eye by Sirius Scheimpflug Analyzer

The corneal aberrometry evaluated by Sirius Scheimpflug Analyzer showed that RE total root mean square (RMS) of High order aberrations (HOA) was 1.98 um, RMS coma was 1.56 um and RMS astigmatism was 1.95 um while LE showed RMS HOA of 0.68 um, RMS coma of 0.60 um and RMS astigmatism of $0.95 \mathrm{um}$. Despite the advanced stage of keratoconus with very steep cornea the refraction was of a low degree with spherical equivalent $(\mathrm{SE})$ in $\mathrm{RE}=-2.25$,
+0.75 in LE). The discrepancy between the refraction and the corneal keratometry power made the evaluation of the case relatively challenging. Keratoconus in hyperopia was our provisional diagnosis and to confirm it; axial length was measured in both eyes. The axial length of the RE was $19.63 \mathrm{~mm}$, fig (2), while the axial length of the LE was 19.97 $\mathrm{mm}$. The anterior chamber showed length of $\mathrm{RE}=2.86 \mathrm{~mm}$ and $\mathrm{LE}=2.85 \mathrm{~mm}$. 


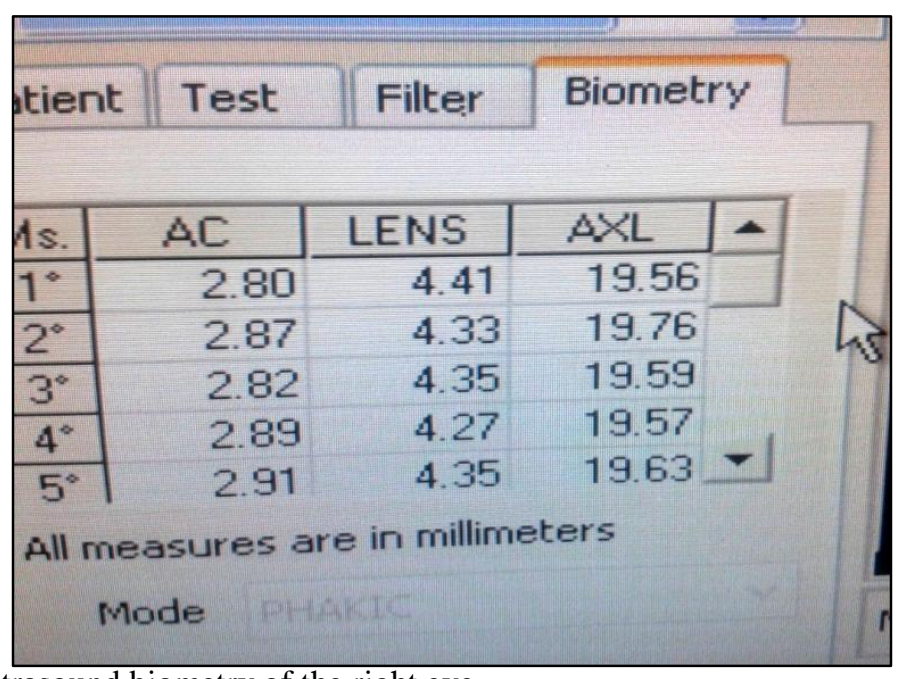

Figure (2) a-scan ultrasound biometry of the right eye

The decision was to do right simultaneous intracorneal ring segments implantation with KeraRings (Mediphacos, Belo Horizonte, Brazil), followed by corneal collagen crosslinking in the same session. The tunnel for rings was created with femtosecond laser; Advanced Femtosecond Laser (iFS, Abbott). Femtosecond laser parameters for the corneal tunnel were: inner diameter: $5 \mathrm{~mm}$, outer diameter: $5.9 \mathrm{~mm}$, depth: $80 \%$ of thinnest central corneal thickness, incision site: at the axis of the steepest corneal meridian and energy $2.00 \mathrm{~mJ}$. The Kerarings were implanted according to the Keraring nomogram rules. At the same session, transeptithelial accelerated corneal collagen cross linking was done by The KXL ${ }^{\circledR}$ System accelerated CXL (Avedro). The corneal surface was treated by the application of $0.25 \%$ riboflavin solution supplemented with BAC, EDTA, trometamol, hydroxypropyl-methylcellulose (ParaCell, Avedro) for $4.50 \mathrm{~min}$, and
$0.25 \%$ riboflavin solution (VibeX Extra, Avedro) for $6 \mathrm{~min}$. Drops were applied every $90 \mathrm{~s}$ during the soak time. This was followed by 5.20 minutes accelerated CXL using the pulsed mode with $45 \mathrm{~mW} /$ CC power without corneal epithelial debridement. One drop of VibeX Extra was applied every $90 \mathrm{~s}$ during irradiation. Postoperative medication included topical antibiotics (Gatifloxacin 0.3\% 5 times/day for one week), Topical steroid (Prednisolone acetate $1 \% 5$ times/day for one week), Lubricant eye drops and systemic nonsteroidal anti-inflammatory drugs. UCVA, BCVA, sphere, cylinder, spherical equivalent, RMS coma, RMS high order aberrantions and RMS astigmatism were measur ed postoperatively in the first week, 1 month, 3 month, 6 month. Table (1), Figure (3) written informed consent of the patient was obtained, and patient anonymity was preserved.

Table (1) Pre and postoperative data with follow up period of 6 months

\begin{tabular}{|l|l|l|l|l|l|l|}
\hline & Preoperative & 1 week & 1 month & 3 month & 6 month \\
\hline UCVA & 1.3 & 1.00 & 0.78 & 0.6 & 0.78 \\
\hline BCVA & 0.78 & 0.6 & 0.48 & 0.48 & 0.48 \\
\hline Sphere & -0.50 & +1.75 & +1.00 & +1.25 & +1.00 \\
\hline Cylinder & -3.50 & -2.00 & -2.25 & -1.75 & -1.75 \\
\hline Spherical equivalent & -2.25 & +0.75 & -0.125 & +0.375 & +0.125 \\
\hline RMS Coma & 1.98 & 0.94 & 0.96 & 0.95 & 1.10 \\
\hline RMS HOA & 1.56 & 0.97 & 0.98 & 0.96 & 0.98 \\
\hline RMS Astigmatism & 1.95 & 1.93 & 1.85 & 1.91 & 1.94 \\
\hline Flat K & 52.59 & 48.48 & 48.50 & 48.61 & 48.65 \\
\hline Steep K & 55.28 & 50.59 & 50.62 & 50.63 & 50.82 \\
\hline
\end{tabular}




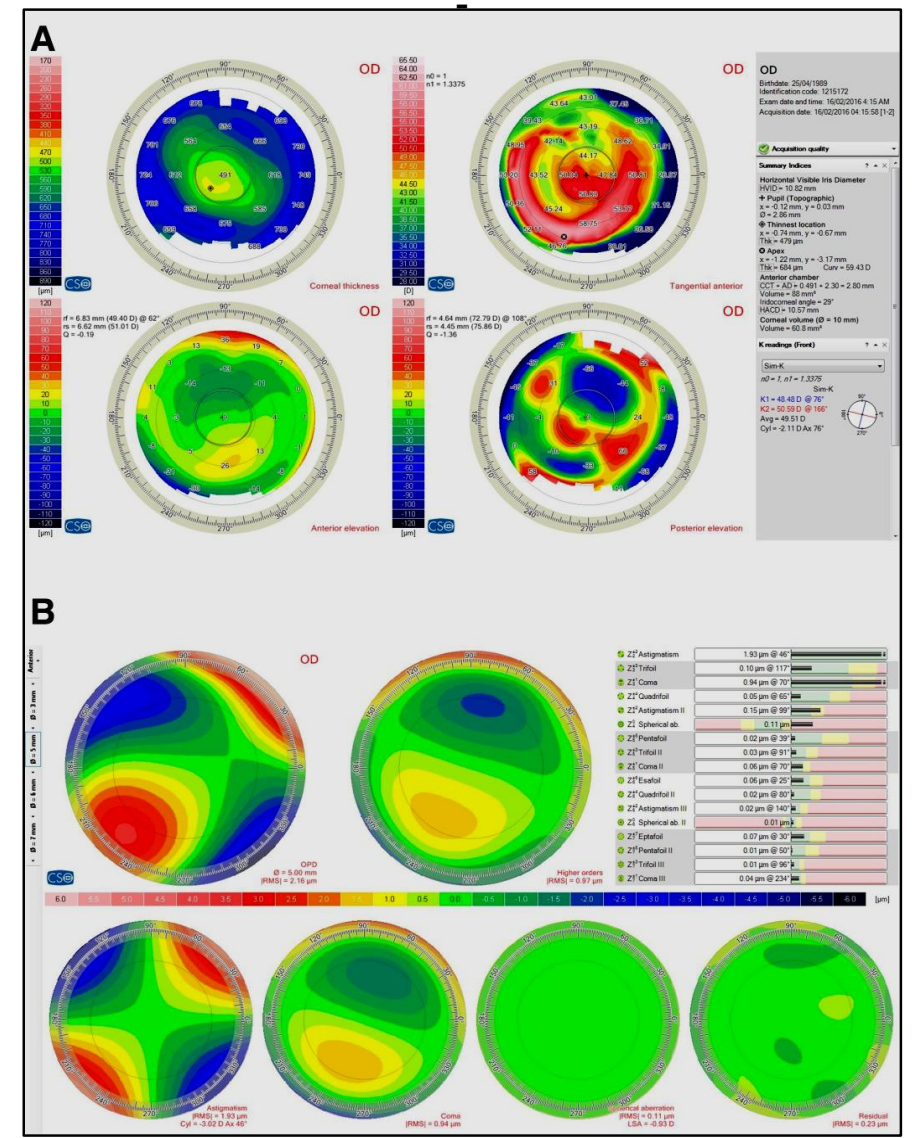

Figure (3) A) the postoperative corneal topography and B) corneal aberrometry of the right eye by Sirius Scheimpflug Analyzer.

\section{Discussion}

In our case, although, the keratoconus in the right eye was grade 3 (Maximum K 56 Ds with posterior elevation of $45 \mu \mathrm{m}$ ), the manifest refraction was of a low spherical component that could only be explained by short axial length eye $(19.63 \mathrm{~mm})$. As keratoconus is known for its myopic component, its relationship to axial myopia was controversial along the literature [6-8]. The myopia was mostly attributed to curvature myopia due to the ecstatic and steep cornea. Yet Ernst et al [9] showed a statistically increase in axial length in keratoconic eyes vs emmetropes $(24.40 \mathrm{~mm}$ vs. $23.24 \mathrm{~mm}$ ). The occurrence of keratoconus along with hyperopia mostly seems outside our scope of thinking and it has been scarcely mentioned in literature $[10,11]$. The fact that we don't have old glass prescriptions of the patient made our diagnosis of already existing hyperopia theoretical and needed confirmation with axial length.
The measurement of axial length revealed a short axial length (under $20 \mathrm{~mm}$ ). So our explanation was that low refractive error is due to the fact of shifting of hyperopia before into the presenting low refractive error. We decided to do corneal collagen cross linking due to the relatively young age of the patient. But the argument was whether to implant intracorneal stromal rings (ICSR) to induce flattening (which may lead to hyperopic shift in this already low refraction with short globe) or not. ICRSs main effect is central corneal flattening which leads to decrease in both myopia and astigmatism induced by keratoconus. Also kerarings implanted by femtosecond laser had been reported to be associated with a reduction of corneal aberration [12]. We decided to go for both femtosecond ICSRS with Epi-on accelerated CXL simultaneously after counseling the patient and knowing that she is intolerant to either glasses or contact 
lenses. Six months of follow up showed favorable results with high patient satisfaction. There was improvement in UCVA, BCVA, with mild hyperopic shift in the sphere as expected with mild decrease in the cylinder, and induced corneal flattening (decreased K1, K2). As for the corneal aberrations, improvement was remarkable especially in coma (decreased by $0.90 \mathrm{um}$ at the 6 month), in HOA (decreased by $0.58 \mathrm{um}$ at the 6 month) with no change in RMS Astigmatism. These results were in agreement with Piñero et al, [13] who found that high order aberrations, coma and astigmatism decreased after kerarings implantation using femtosecond laser tunnel creation.

\section{Conclusion}

Management of keratoconus with low error and short axial length globe shouldn't depend on refraction only but should take into consideration the grade of keratoconus, and aberrometric error. The main role of ICSRs in such cases is to effectively decrease HOA in these patients thus achieving better visual quality.

\section{References}

1. Bayraktar Bilen, N., Hepsen, I., Arce, C. Correlation between visual function and refractive, topographic, pachymetric and aberrometric data in eyes with keratoconus. Int J Ophthalmol 2016; 9(8) $: 1127-1133$

2. Doyle, S., Bullock, J., Gray, C., Spencer, A., Cunningham, C. Emmetropisation, axial length, and corneal topography in teenagers with Down's syndrome. Br J Ophthalmol 1998; 82 (7): 793-796.

3. Giacomin, N., Mello, G., Medeiros, C., Kiliç, A., Serpe, C., Almeida, H., Kara-Junior, N., Santhiago, M. Intracorneal ring segments implantation for corneal ectasia. $\boldsymbol{J}$ Refract Surg 2016; 32 (12): 829-839.

4. Kanellopoulos, A., Pe, L., Perry, H., Donnenfeld, E. Modified intracorneal ring segment implantations (INTACS) for the management of moderate to advanced keratoconus: efficacy and complications. Cornea 2006; 25 (1): 29-33.

5. Alio, J., Shabayek, M., Artola, A. Intracorneal ring segments for keratoconus correction: long-term follow-up. J Cataract Refract Surg 2006; 32 (6): 978-985.

6. Güneş, A., Keratoconus association with axial myopia. Turk J. Ophthalmol 2012; 42: 246-248
7. Touzeau, O., Allouch, C, Borderie, V., Laroche, L. The relat-ionship between keratoconus and axial myopia. $\boldsymbol{J} \boldsymbol{F r}$ Ophthalmol 2004; 27: 765-771.

8. Lanier, J., Bullington, R., Prager, T. Axial length in keratoconus. Cornea 1992; 11 (3): 250-254.

9. Ernst, B., Hsu, H. Keratoconus association withaxial myopia: A prospective biometric study. Eye Contact Lens 2011; 37 (1): 2-5

10. Martin, R. Keratoconus with high hyperopia. Eye Contact Lens 2009; 35 (3): 159-162.

11. Abad, J., Awad, A., Kurstin, J. Hyperopic keratoconus. J Refract Surg 2007; 23 (5): 520-523.

12. Seleet, M., Soliman, A., Alaaeldin, O. Femt-osecond laser intracorneal ring segment implantation based on a nomogram modification in type 1 and type 2 ectasia. J Egypt Ophthalmol Soc 2015; 108 (1): 1-5.

13. Piñero, D., Alio, J., El Kady, B, Coskunseven, E., Morbelli, H., UcedaMontanes, A., Maldonado, M., Cuevas, D., Pascual, I. Refractive and aberrometric outcomes of intracorneal ring segments for ker-atoconus: mechanical versus femtosecond-assisted procedures. Ophthalmology 2009; 116 (9): 1675-1687. 